\title{
Back from the dead: reanimation following severe hypothermic cardiac arrest
}

Konstantinos Alexopoulos (Meds 2016)

Faculty Reviewer: Dr Ramiro Arellano, MD, MSc, FRCPC (Department of Anesthesia and Perioperative Medicine)

\section{BACKGROUND}

Hypothermia is clinically defined as a drop in core body temperature below $35^{\circ} \mathrm{C}$ in a previously healthy individual in the absence of pre-existing inherent underlying thermoregulatory pathology. Classification of hypothermia below $35^{\circ} \mathrm{C}$ is described as mild $\left(32^{\circ} \mathrm{C}\right.$ to $\left.35^{\circ} \mathrm{C}\right)$ and moderate $\left(28^{\circ} \mathrm{C}\right.$ to $\left.32^{\circ} \mathrm{C}\right)$. A temperature below $28^{\circ} \mathrm{C}$ is classified as severe. At $28^{\circ} \mathrm{C}$ cardiopulmonary function is likely to become compromised. The myocardium becomes vulnerable to arrhythmia, ventricular asystole and subsequently complete cardiovascular collapse. Concomitant cognitive impairment is most commonly associated with severe hypothermia and cold injury. These patients tend to be individuals with mental illness, homelessness, and alcohol and illicit drug use. Recreational accidents such as skiing comprise the second most common group for hypothermic injury. ${ }^{1}$

There has been much academic debate and speculation regarding the optimal approach to rewarming any patient suffering from hypothermia. ${ }^{2}$ Mild hypothermia responds well to external rewarming techniques like forced heated air blankets. ${ }^{3}$ Moderate hypothermia is more precarious from a physiological standpoint. The myocardium is exceptionally vulnerable at these temperatures. Consequently, rapid rewarming techniques are favoured. This includes warmed intravenous (IV) fluids and warm peritoneal lavage in those who have not demonstrated overt signs of cardiac instability. ${ }^{4}$ Less commonly, these modalities can be augmented with airway warming and pleural irrigation. ${ }^{4,5}$

If patients with severe hypothermia have a sustainable cardiac rhythm, they are at increased risk for cardiovascular collapse. ${ }^{6}$ The optimal modality for rewarming a patient with severe hypothermic cardiac arrest remains a highly controversial topic. ${ }^{7}$ The most recent retrospective comparative study in 68 patients demonstrated cardiopulmonary bypass (CPB) rewarming to be far superior to conventional methods of rewarming, with mortality rates of $15.8 \%$ and $53.3 \%$, respectively. Reanimation on CPB allows for several advantages, including rapid rewarming, but most importantly oxygenation and perfusion are preserved. A retrospective study analyzed the long-term neurological outcomes of survivors who reported normal neuropsychological findings in $93.3 \%$ of cases and normal brain magnetic resonance imaging in $86.7 \%$ of cases. ${ }^{8}$

\section{CASE PRESENTATION}

Patient MG is a 29-year-old gentleman with no previous medical history who presented to the emergency department in a peripheral hospital. MG was found near his vehicle which had crashed into a nearby tree. His core temperature was $27^{\circ} \mathrm{C}$. There was minimal damage to the vehicle. He reportedly left his partner's home at midnight and had been drinking alcohol and consuming illicit drugs.

The patient had been intubated on scene and peripheral vascular access obtained. Cardiopulmonary resuscitation (CPR) had also been initiated he before he was transferred to hospital. CPR was continued in conjunction with external rewarming techniques which proved futile. The physician in the periphery contacted CritiCall Ontario and was put in contact with the nearest facility with $\mathrm{CPB}$ capabilities and a cardiac surgery team. The decision was made to transfer MG to University Hospital (UH) for reanimation on bypass. Throughout the resuscitative efforts, MG remained asystolic, with a Glasgow Coma Scale (GCS) score of 3 . His neurological exam at that time showed dilated pupils with a normal Babinski sign. No other obvious injuries were appreciated on secondary survey.

Upon arrival to UH, MG was taken directly to the operating room (OR). Core temperature was repeated and confirmed at $21^{\circ} \mathrm{C}$. The patient was switched from manual respiration to mechanical ventilation. He had elevated airway pressures and was difficult to ventilate. IV access was in situ and a radial arterial line and right internal jugular vein triple lumen central line were placed under ultrasound guidance. Rocuronium $50 \mathrm{mg}$ IV was administered for paralysis along with 40000 units of heparin. Initial arterial blood gas (ABG) showed pH 6.15, $\mathrm{pCO}_{2} 67 \mathrm{mmHg}, \mathrm{pO}_{2} 431 \mathrm{mmHg}$, bicarbonate $12 \mathrm{mmol} / \mathrm{L}$, sodium $143 \mathrm{mmol} / \mathrm{L}$, potassium of $7.5 \mathrm{mmol} / \mathrm{L}$, lactate $14.5 \mathrm{mmol} / \mathrm{L}$.

Concurrently, the surgery team attempted a cutdown of the right groin, but the patient's right femoral artery could not be cannulated using a Seldinger technique. This was likely due to truncation and collapse proximally. Therefore, the left femoral artery was cut down and cannulated. $10 \mathrm{mg}$ of midazolam was administered and $\mathrm{CPB}$ was initiated.

MG was successfully rewarmed to $35^{\circ} \mathrm{C}$ and his cardiac rhythm showed ventricular fibrillation. MG was cardioverted once externally and converted to a normal sinus rhythm at a rate of 78 beats per minute with a mean arterial pressure (MAP) of $65 \mathrm{mmHg}$. ABG at that time showed $\mathrm{pH} 7.15, \mathrm{pCO}_{2} 39 \mathrm{mmHg}, \mathrm{pO}_{2} 472 \mathrm{mmHg}$, bicarbonate $12 \mathrm{mEq} / \mathrm{L}$, sodium $145 \mathrm{mmol} / \mathrm{L}$, potassium of $6.4 \mathrm{mmol} / \mathrm{L}$, lactate $>15 \mathrm{mmol} / \mathrm{L}$. He initially required no vasopressor support.

Following cardioversion and weaning from CPB, MG's airway pressures remained high on the anesthesia machine. Pink froth began to emanate from the endotracheal tube and around the patient's mouth. Videolaryngoscopy (Glidescope) showed the endotracheal tube between the vocal cords, and it was determined that the endotracheal tube cuff had ruptured. The patient was suctioned and reintubated using the Glidescope with an $8.0 \mathrm{~mm}$ internal diameter 
endotracheal tube. A ventilator from the medical-surgical intensive care unit (MSICU) was employed with little improvement in ventilation or airway pressures. Solumedrol $125 \mathrm{mg}$ IV along with furosemide $40 \mathrm{mg}$ IV was administered. There was mild improvement in ventilation. At this point in time, norepinephrine was started at 4 $\mu \mathrm{g} / \mathrm{min}$ for hemodynamic support.

A transesophageal echocardiography (TEE) probe was inserted to assess heart function. The examination overall revealed no gross wall motion abnormalities, and the left ventricular function was mildly globally depressed. Given the ABG results and frothing from the tube, which constituted acute noncardiogenic pulmonary edema, the decision was made to reinitiate CPB for exploratory laparotomy. Intraoperative physical exam had revealed that MG's abdomen was becoming quite distended and tense. General surgery was consulted and performed an exploratory laparotomy with no acute findings. The abdomen was left open with an ABThera vacuum dressing in place.

Following the laparotomy, the decision was made to convert the $\mathrm{CPB}$ circuit to an extracorporeal membrane oxygenation (ECMO) circuit and transfer the patient to the cardiac surgery recovery unit (CSRU). A venovenous ECMO circuit was considered. However, given MG's extended period of cardiac arrest, an arteriovenous (AV) circuit was placed. He remained on the norepinephrine infusion at $8 \mu \mathrm{g} / \mathrm{min}$ and after conversion to ECMO his ABG showed pH 7.31, $\mathrm{pCO}_{2} 41 \mathrm{mmHg}, \mathrm{pO}_{2} 263 \mathrm{mmHg}$. Throughout the evening his vasopressor requirements increased and other injuries began to present themselves.

In the coming weeks, MG developed compartment syndrome in all limbs. Despite fasciotomy and surgical management, he required amputation of his right leg above the knee and his left arm above the elbow. Despite all of the sequelae from the initial hypothermic insult, MG regained neurological function with no focal neurological deficits. MG survived his extended stay in the CSRU and MSICU and was discharged to a rehabilitation facility.

\section{DISCUSSION}

This case presented some very unique challenges from a surgical and anesthetic management perspective. The case was treated as a trauma and the principles of resuscitation were employed at all levels of care. However, it is apparent that access to tertiary and quaternary hospitals is paramount to successful resuscitation. Had this gentleman remained in the periphery, he would likely not have survived. Despite the obvious morbidity MG faces with his quality of life being impacted by his amputations, he remained neurologically intact.

His airway and ventilation were of major concern throughout the reanimation. Despite correct placement of the endotracheal tube, it is likely that the aggressive resuscitative efforts increased pressure around the cuff of the endotracheal tube. More importantly the development of noncardiogenic pulmonary edema/acute respiratory distress syndrome (ARDS) upon rewarming posed significant management issues. This has been described in the literature upon rewarming of hypothermic patients. ${ }^{9}$ At this point in time there has been no trial which advocates for a specific treatment modality. Positive pressure ventilation in severe ARDS with IV steroids and diuretics are the mainstay of treatment. AV ECMO was preferred as this would permit bypass of the lungs entirely so that tissue integrity might be preserved during convalescence.

Furthermore, the patient presented with a profound acidosis with an associated hyperkalemia of $7.5 \mathrm{mmol} / \mathrm{L}$. The potassium level prior to initiating CPB was associated with greater mortality for the patient. This is likely due to the fact that in mild hypothermia there is an initial inward shift of potassium, which is likely contributory to the arrhythmias that develop in those with moderate hypothermia. As the tissue becomes hypoperfused during cardiac arrest, cell necrosis will elevate the serum potassium. ${ }^{10,11}$ Profound hyperkalemia is a grave prognostic indicator as mentioned earlier on. Experts in reanimation have considered a potassium level greater than $10 \mathrm{mmol} / \mathrm{L}$ as the point of no return ${ }^{12}$ and subsequently a decision is made to withhold reanimation on CPB in that population.

Neurologically the patient did remarkably well considering his downtime was anywhere from 6 to 12 hours. Following deep hypothermic cardiac arrest, the emergent use of CPB has become advocated for as the preferred method of rewarming. It has shown superiority to conventional rewarming methods, particularly in adult patients. The survival benefits and reduced morbidity with the use of CPB in hypothermic arrest is significant. However, there has been no trial which compares outcomes in a randomized manner. ${ }^{2}$ Therefore, management is left up to the discretion of the trauma team in consultation with cardiac surgery and anesthesia. One thing remains certain: it has been said that those who work and live in extreme northern climates say, "You're not dead until you're warm and dead."

\section{REFERENCES}

1. Vretenar DF, Urschel JD, Parott JCW, Unruh MW. Cardiopulmonary bypass resuscitation for accidental hypothermia. Ann Thorac Surg 1994; 58:895-8.

2. Sepehripour AH, Gupta S, Lall, KS. When should cardiopulmonary bypass be used in the setting of severe hypothermic cardiac arrest? Interactive Cardiovascular and Thoracic Surgery 2013; 17:564-570

3. Jolly BT, Ghezzi KT. Accidental hypothermia. Emerg Med Clin North Am 1992; 10:311-27.

4. Bolgiano E, Sykes L, Barish RA, Zickler R, Eastridge B. Accidental hypothermia with cardiac arrest: recovery following rewarming by cardiopulmonary bypass. J Emerg Med 1992;10:427-33.

5. Hall KN, Syverud SA. Closed thoracic cavity lavage in the treatment of severe hypothermia in human beings. Ann Emerg Med 1990; 19:204-6.

6. Wolpoth BH, Locher T, Leupi F, Schupbach P, Muhlemann W, Althaus U. Accidental deep hypothermia with cardiopulmonary arrest: extracorporeal blood rewarming in 11 patients. Eur J Cardiothorac Surg 1990; 4:390-3.

7. Schissler P, Parker MA, Scott SJ Jr. Profound hypothermia: value of prolonged cardiopulmonary resuscitation. South Med J 1981; 74:474-7. 
8. Morita S, Inokuchi S, Yamagiwa T, Lizuka S, Yamamoto R, Aoki $\mathrm{H}$ et al. Efficacy of portable and percutaneous cardiopulmonary bypass rewarming versus that of conventional internal rewarming for patients with accidental deep hypothermia. Crit Care Med 2011; 39:1064-8.

9. Morales CF, Strollo PJ. Noncardiogenic pulmonary edema associated with accidental hypothermia. Chest 1993; 103: 971-3.

10. Hauty MG, Esrig BC, Hill JG, Long WB. Prognostic factors in severe accidental hypothermia: experience from the Mt. Hood tragedy. J Trauma 1987;27:1107-11.
11. Sprung J, Cheng EY, Gamulin S, Kampine JP, Bosnjak ZJ. The side effect of acute hypothermia and serum potassium concentration on potassium cardiotoxicity in anesthetized rats. Acta Anaesthesiol Scand 1992; 36: 825-30.

12. Koht A. Cane R. Cerullo JD. Serum potassium levels during prolonged hypothermia. Intensive Care Med 1983; 9:275-7. 